\title{
Spacetime is entangled in a Bell state
}

\author{
Open Physics Collaboration*†
}

February 29, 2020

\begin{abstract}
We propose a connection for the Minkowski's invariant proper time and one of the Bell states.
\end{abstract}

keywords: special relativity, invariant proper time, quantum Bell states

\section{Preamble}

1. The views of space and time which I wish to lay before you have sprung from the soil of experimental physics, and therein lies their strength. They are radical. Henceforth space by itself, and time by itself, are doomed to fade away into mere shadows, and only a kind of union of the two will preserve an independent reality. [1]

Hermann Minkowski

*All authors with their affiliations appear at the end of this paper.

${ }^{\dagger}$ Corresponding author: mplobo@uft.edu.br| Join the Open Physics Collaboration 


\section{Proper Time}

2. Minkowski showed that space and time are inseparable and unified by a unique and invariant quantity called the proper time $(\tau)[1,2]$.

3.

$$
\tau^{2}=t^{2}-x^{2}
$$

4. $t$ is the time interval from two events in a given inertial frame.

5. $x$ is the space interval from two events in a given inertial frame.

\section{Spacetime entanglement}

6. One of the Bell states is

$$
|\psi\rangle=\frac{1}{\sqrt{2}}(|00\rangle-|11\rangle),
$$

where $|0\rangle$ and $|1\rangle$ are quantum states.

7. Note the resemblance between (3) and (6).

8. One can assume that quantum spacetime is entangled in the following Bell state

$$
|\tau \tau\rangle=\frac{1}{\sqrt{2}}(|t t\rangle-|x x\rangle) .
$$

9. $|t\rangle$ is the quantum state that originates time in the classical scale.

10. $|x\rangle$ is the quantum state that originates space in the classical scale.

11. This notation suggests the following tensor products.

12.

$$
|\tau \tau\rangle \equiv|\tau\rangle_{1}|\tau\rangle_{2} \equiv|\tau\rangle_{1} \otimes|\tau\rangle_{2}
$$

13.

$$
|x x\rangle \equiv|x\rangle_{1}|x\rangle_{2} \equiv|x\rangle_{1} \otimes|x\rangle_{2}
$$


14.

$$
|t t\rangle \equiv|t\rangle_{1}|t\rangle_{2} \equiv|t\rangle_{1} \otimes|t\rangle_{2}
$$

15. $|x\rangle_{1}$ and $|x\rangle_{2}$ are the entangled quantum states of position in different regions.

16. Similarly, $|t\rangle_{1}$ and $|t\rangle_{2}$ are the entangled quantum states of time in different periods.

\section{Momentum and energy}

17. A similar approach can be done with the magnitude of momenergy [2],

$$
E^{2}-\vec{p}^{2}
$$

which is Lorentz invariant.

\section{Final Remarks}

18. Time and space can be assembled as a qubit in the very quantum realm, like in the Planck scale, for instance.

19. A lot of further investigation need to be done regarding (8).

20. Other discussions on time that are related to this article can be found here $|3-5|$.

\section{Open Invitation}

Review, add content, and co-author this paper [6,7]. Join the Open Physics Collaboration. Send your contribution to mplobo@uft.edu.br. 


\section{Ethical conduct of research}

This original work was pre-registered under the OSF Preprints [8], please cite it accordingly [9]. This will ensure that researches are conducted with integrity and intellectual honesty at all times and by all means.

\section{References}

[1] Minkowski, H. "The union of space and time", 1908.

[2] Taylor, Edwin F., and John Archibald Wheeler. Spacetime Physics. Macmillan, 1992.

[3] Lobo, Matheus P. "The Inner Bound of Quantum Spacetime." OSF Preprints, 11 June 2019. https://doi.org/10.31219/osf.io/6zf3n

[4] Lobo, Matheus P. "Spacetime Pouring." OSF Preprints, 21 May 2019. https://doi.org/10.31219/osf.io/zwfb5

[5] Lobo, Matheus P. "Time Travel: Coexistence of Past, Present, and Future?" OSF Preprints, 2 Sept. 2019. https://doi.org/10.31219/osf.io/7ruay

[6] Lobo, Matheus P. "Microarticles." OSF Preprints, 28 Oct. 2019. https://doi.org/10.31219/osf.io/ejrct

[7] Lobo, Matheus P. "Simple Guidelines for Authors: Open Journal of Mathematics and Physics." OSF Preprints, 15 Nov. 2019. https://doi.org/10.31219/osf.io/fk836

[8] COS. Open Science Framework. https://osf.io

[9] Lobo, Matheus P. "Spacetime Is Entangled in a Bell State." OSF Preprints, 27 Nov. 2019. https://doi.org/10.31219/osf.io/xc4ys 


\section{The Open Physics Collaboration}

Matheus Pereira Lobo (lead author, mplobo@uft.edu.br),,2

João Marcos Costa da Silva, ${ }^{1}$ Suel Sousa Ribeiro ${ }^{1}$

${ }^{1}$ Federal University of Tocantins (Brazil); ${ }^{2}$ Universidade Aberta (UAb, Portugal) 